\title{
Survey on Fast and Intelligent Deep Web Crawler Using Machine Learning Approach
}

\author{
Kalyani Thodage \\ ME Student, Department of Computer Engineering, Sinhgad Academy of Engg. Pune, Maharashtra, India
}

\begin{abstract}
The quantity of site pages accessible in the Internet is developing enormously every day. For this situation seeking significant data in the Internet is hard errand. A great deal of this data is holed up behind question frames that interface to unexplored databases containing brilliant organized information. Conventional web crawlers can't get to and list this concealed a portion of the Web, recovering this shrouded data is testing assignment. Consequently, we propose a two-stage structure, to be specific Smart Crawler, for successfully reaping profound web interfaces. In the first stage that is site finding, focus pages are sought with the assistance of internet searchers which thus abstain from going by an extensive number of pages. To accomplish more exact results for an engaged slither, Smart Crawler positions sites to organize exceptionally important ones for a given point. In the second stage, versatile connection excavating so as to position accomplishes quick in-site seeking most significant connections.
\end{abstract}

Keywords: Deep web Crawler, Adaptive learning, Form Classifier, Ranker

\section{Introduction}

Basically, which means of crawler is creeps around the ground. In web slithering, the crawler creeps around the pages, assembles and classifies data on the World Wide Web. The crawler contains of three sections: First is the insect, additionally called as crawler. The bug visits the pages, gets the data and after that takes after the connections in different pages inside of a site. The creepy crawly comes back to crept site over normal interim of time. The data found in the first stage will be given to the second stage, the list. It is likewise understood as inventory. The file is similar to a database, containing each duplicate of page that crawler finds. In the event that a site page changes then the duplicate is redesigned with new data in the database. Third part is programming. This is a system that filters a huge number of website pages recorded in the file to discover matches to inquiry and level them all together of what it accepts as generally important.

It is troublesome assignment to find profound web interfaces, in light of the fact that they are not recorded by any web indexes. They are typically once in a while dispersed and keep continually evolving. To manage above issue, past work has proposed two sorts of crawlers which are non specific crawlers and centered crawlers. Non specific crawler brings all the searchable structures and don't concentrate on a particular point where as Focused crawlers are the crawler which concentrates on a particular theme. Structure centered crawler (FFC)[2] and Adaptive crawler for shrouded web passages (ACHE)[3] plans to productively and consequently distinguish different structures in the same space. The fundamental segments of FFC are connection, page, structure classifiers and boondocks director for centered creeping of web-structures. Throb amplifies the engaged technique of FFC with extra segments as structure separating and versatile connection learner. The precision of centered crawlers is low as far as recovering significant structures. Profound web [1],[4] likewise called as dim web or undetectable web. Profound web[5] are the substance on the web which is not filed in an internet searcher. It is an accumulation of sites that are openly accessible however conceal the IP locations of a server that keep running on them. Along these lines they can be gone to by the client, however it is hard to figure out who are behind those destinations. Profound web is something you can't situate with a solitary hunt.

A structure for proficiently reaping profound web named Smart Crawler is composed in this paper. Savvy Crawler performs a propelled level of information examination and information extricated from the web. The Smart Crawler is partitioned into two stages:

Site finding and in-site investigating. In the first stage, Smart Crawler performs site-based looking for focus pages with the assistance of web crawlers, abstaining from going to an expansive number of pages. To accomplish more point by point results for an engaged creep, Smart Crawler positions sites to organized very pertinent once for a given subject. In the second stage, Smart Crawler accomplishes quick in-site looking to uncover most important connections with a versatile connection positioning.

Site finding strategy uses opposite looking procedure and incremental two-level site positioning system for uncovering applicable locales and to accomplish more information sources. Amid the in-webpage investigating stage, a connection tree is intended for adjusted connection organizing, killing predisposition toward pages in prevalent indexes.

\section{Related Work}

There are numerous writing in the territory of web crawlers. In late 1994, The RBSE (Repository Based Software Engineering venture first dispatch the Web Crawler in light of two projects: first was "creepy crawly", it keep up a line in a social database, and second was "vermin", it is an adjusted www ASCII program that download the pages from web[6]. At that point the second WebCrawler was freely accessible full-content list of a subset of the web which depended on lib-WWW to download pages, and other system to parse and

\section{Volume 4 Issue 11, November 2015}




\section{International Journal of Science and Research (IJSR) \\ ISSN (Online): 2319-7064}

Index Copernicus Value (2013): 6.14 | Impact Factor (2014): 5.611

arrange URLs for expansiveness first investigation of web diagram.

Locating deep web content sources- A late study demonstrates that the harvest rate of profound web is low just 647,000 particular web structures were found by inspecting 25 million pages from the Google list (around $2.5 \%)$. Nonexclusive crawlers are for the most part created for portraying profound web and index development of profound web assets, that don't farthest point seek on a particular theme, however endeavor to bring every single searchable structure. The Database Crawler in the Meta Queries is intended for consequently finding question interfaces. Database Crawler first discovers root pages by an IP-based testing, and afterward performs shallow slithering to creep pages inside of a web server beginning from a given root page. The IP based examining overlooks the way that one IP location may have a few virtual hosts, along these lines missing numerous site.

\section{A. Internet archive Crawler \\ Mike Burner outlined the Internet Archive Crawler [7] was the first paper that concentrated on the difficulties brought on by the size of web. It utilizes various machine to slither the web and it creep on 100 million URLs[6]. Every crawler procedure read a rundown of seed URLs for its relegated locales from plate into per-site line, and after that it utilizes nonconcurrent $\mathrm{I} / \mathrm{O}$ guidelines to get pages from these lines in parallel. It has additionally manage the issue of changing DNS records, so it keeps the authentic document of hostname to IP mapping.}

\section{B. Google Crawler}

The first Google slithering framework comprise of a five creeping parts which was running in different process and download the pages [7].

Every crawler procedure utilized nonconcurrent I/O guidelines to get the information from up to 300 web servers in parallel. At that point every one of the crawlers transmit downloaded pages to a solitary Store Server handle that compacted the page and store them on disk[6]. Google Crawler depended on $\mathrm{C}++$ and Python. This crawler was incorporated with the indexing procedure( content parsing was finished full-content indexing furthermore for URL extraction).

\section{Mercator Web Crawler}

Heydon and Najork present a web crawler which was profoundly adaptable and effectively extensible [8][6]. It was composed in Java. The main rendition was non-disseminated and later the circulated form was made accessible which split up the URL space over the crawlers as indicated by host name and keep away from the potential bottleneck of an incorporated URL server.

\section{Web Fountain crawler}

Another appropriated and secluded crawler spoke to by IBM[8][6]. It has three noteworthy part, Multi strung slithering procedures, copy substance and focal controlled procedure in charge of doling out work. It was composed in $\mathrm{C}++$ and utilized MPI to encourage the correspondence between the different procedure. It was conveyed on a bunch of 48 slithering machine.

\section{E. IRLbot Web crawler}

As of late, Yan et al. depict IRLbot, which is single procedure web crawler [6]. It has the capacity scale to amazingly substantial web accumulation without execution corruption. It slither more than two month and downloads the 6.4 billion website pages.

\section{Proposed Work}

In this paper Smart Crawler contain a novel two-stage system to address the issue of scanning for concealed web assets.

In any case, to enhance exactness of structure classifier, prequestion and post-inquiry approaches for characterizing profound web structures are joined. Moreover, the connections in these pages are separated into Candidate Frontier. To organize joins in Candidate Frontier, Smart Crawler positions them with Link Ranker. At the point when the crawler finds another site, the site's URL is embedded into the Site Database. The Link Ranker is adaptively enhanced by an Adaptive Link Learner, which gains from the URL way prompting pertinent structures.

\section{Feature Selection and Ranking}

SmartCrawler experiences an assortment of site pages amid a slithering procedure and the way to productively creeping and wide scope is positioning distinctive locales and organizing connections inside of a site. This segment first talks about the online element development of highlight space and versatile learning procedure of SmartCrawler, and after that depicts the positioning instrument.

\subsection{Online Construction of Feature Space-}

In Smart Crawler, examples of connections to applicable destinations and searchable structures are found out online to assemble both webpage and connection rankers. The capacity of web learning is imperative for the crawler to maintain a strategic distance from predispositions from introductory preparing information and adjust to new examples.

The feature space of deep web sites (F SS) is de- fined as:

$$
\mathrm{F} \mathrm{SS}=\{\mathrm{U}, \mathrm{A}, \mathrm{T}\}
$$

where $\mathrm{U}, \mathrm{A}, \mathrm{T}$ are vectors corresponding to the feature context of URL, anchor, and text around URL of the deep web sites.

The feature space of links of a site with embedded forms ( $\mathrm{F}$ SL) is defined as:

$$
\mathrm{F} \mathrm{SL}=\{\mathrm{P}, \mathrm{A}, \mathrm{T}\},
$$

where $\mathrm{A}$ and $\mathrm{T}$ are the same as defined in F SS and P is the vector related to the path of the URL, since all links of a specific site have the same domain.

Each feature context can be represented as a vector of terms with a specific weight. The weight $\mathrm{w}$ of term $\mathrm{t}$ can be defined as: 


\section{International Journal of Science and Research (IJSR) \\ ISSN (Online): 2319-7064}

Index Copernicus Value (2013): 6.14 | Impact Factor (2014): 5.611

\section{wt, $\mathrm{d}=1+\log \mathrm{tft}, \mathrm{d}$,}

where $\mathrm{tft}$, $\mathrm{d}$ denotes the frequency of term $\mathrm{t}$ appears in document $\mathrm{d}$, and $\mathrm{d}$ can be $\mathrm{U}, \mathrm{P}, \mathrm{A}$, or $\mathrm{T}$. We use term frequency (TF) as feature weight for its simplicity and our experience shows that TF works well for our application.

\subsection{Adaptive Learning}

Smart Crawler has a versatile learning procedure that redesigns and influences data gathered effectively amid slithering. Intermittently, F SS and F SL are adaptively redesigned to reflect new examples found amid creeping. Therefore, Site Ranker and Link Ranker are redesigned. At long last, Site Ranker re-positions destinations in Site Frontier and Link Ranker overhauls the significance of connections in Link Frontier.

\subsection{Ranking Mechanism}

\subsubsection{Site Ranking}

Smart Crawler positions site URLs to organize potential profound destinations of a given theme. To this end, two elements, site likeness and site recurrence, are considered for positioning. Website comparability measures the theme likeness between another webpage and known profound sites. Site recurrence is the recurrence of a site to show up in different destinations, which demonstrates the fame and power of the site - a high recurrence site is possibly more essential. Since seed destinations are precisely chosen, moderately high scores are doled out to them. Given the landing page URL of another webpage $s=\{\mathrm{Us}, \mathrm{As}, \mathrm{Ts}\}$, the website closeness to known profound sites F SS, can be characterized as takes after: $\mathrm{ST}(\mathrm{s})=\operatorname{Sim}(\mathrm{U}, \mathrm{Us})+\operatorname{sim}(\mathrm{A}$, As) $+\operatorname{sim}(T, T s)$, (4) where capacity $\operatorname{Sim}$ scores the similitude of the related element in the middle of $\mathrm{s}$ and known profound sites. The capacity $\operatorname{Sim}(\bullet)$ is processed as the cosine similitude between two vectors V1 and V2: $\operatorname{Sim}(\mathrm{V} 1, \mathrm{~V} 2)=\mathrm{V} 1 \cdot \mathrm{V} 2|\mathrm{~V} 1|$

\subsubsection{Link Ranking-}

For organizing connections of a site, the connection closeness is registered comparably to the site similitude depicted previously. The distinction incorporates: 1) connection organizing depends on the component space of connections with searchable structures (F SL); 2) for URL highlight $\mathrm{U}$, just way part is considered subsequent to all connections have the same area; and 3 ) the recurrence of connections is not considered in connection positioning. Given another connection $1=\{\mathrm{Pl}, \mathrm{Al}, \mathrm{Tl}\}$, the connection closeness to the element space of known connections with searchable structures F SL is characterized as: $\mathrm{LT}(1)=\operatorname{Sim}(\mathrm{P}$, $\mathrm{Pl})+\operatorname{sim}(\mathrm{A}, \mathrm{Al})+\operatorname{sim}(\mathrm{T}, \mathrm{Tl}),(8)$ where capacity $\operatorname{Sim}(\bullet)$ (Equation 5) scores the comparability of the related component in the middle of 1 and the known in-site joins with structures. At long last, we utilize the connection comparability for positioning distinctive connection.

\section{Form Classifier}

Grouping structures intends to keep structure centered slithering, which sift through non-searchable and unessential structures. For example, an airfare inquiry is frequently cosituated with rental auto and lodging reservation in travel destinations. For an engaged crawler, we have to uproot offpoint look interfaces. Savvy Crawler receives the HIFI technique to channel applicable searchable structures with an organization of basic classifiers. HIFI comprises of two classifiers, a searchable structure classifier (SFC) and an area particular structure classifier (DSFC). SFC is an area autonomous classifier to sift through non-searchable structures by utilizing the structure highlight of structures. DSFC judges whether a structure is point significant or not taking into account the content element of the structure, that comprises of area related terms. The procedure of dividing the element space permits choice of more compelling learning calculations for every element subset The subtle elements of these classifiers are out of the extent of this paper (see [10] for points of interest).

\section{Conclusion}

Smart Crawler accomplishes more exact results by positioning gathered destinations and centering the creeping on a given theme. The in-webpage investigating stage utilizes versatile connection positioning to seek inside of a webpage and configuration a connection tree for wiping out predisposition ward certain registries of a site for more extensive scope of web catalogs.

We have demonstrated that our methodology accomplishes both wide scope for profound web interfaces and keeps up very effective creeping. Savvy Crawler is an engaged crawler comprising of two stages: effective site finding and adjusted in-site investigating. Savvy Crawler performs webpage based situating by contrarily looking the known profound sites for focus pages, which can successfully discover numerous information hotspots for scanty spaces. By focusing so as to position gathered destinations and the slithering on a theme, Smart Crawler accomplishes more precise results. The inwebpage investigating stage utilizes versatile connection positioning to seek inside of a webpage; and we plan a connection tree for wiping out inclination toward specific registries of a site for more extensive scope of web indexes. Our exploratory results on an agent set of areas demonstrate the viability of the proposed two-stage crawler, which accomplishes higher harvest rates than different crawlers. In future work, we plan to consolidate pre-question and postinquiry approaches for ordering profound web structures to further enhance the precision of the structure classifier.

\section{References}

[1] Michael K. Bergman. White paper: The deep web: Surfacing hidden value. Journal of electronic publishing, 7(1), 2001.

[2] Luciano Barbosa and Juliana Freire. Searching for hidden-web databases.In WebDB, pages 1-6, 2005.

[3] Luciano Barbosa and Juliana Freire. An adaptive crawler for locating hidden-web entry points. In Proceedings of the 16th international conference on World Wide Web, pages 441-450. ACM, 2007. 
[4] Right planet's searchable database directory. http://www.completeplanet.com/, 2013.

[5] Y. Wang, T. Peng, W. Zhu, Schema extraction of Deep Web Query Interface", IEEE Transaction On Web Information Systems and Mining, WISM International Conference 2009.

[6] Olston and M .Najork, Web Crawling", Foundations and Trends in Information Retrieval, vol. 4, No. 3 ,pp. 175-246, 2010.

[7] M. Burner, Erawling towards Eternity: Building an Archive of the World Wide Web," Web Techniques Magazine, vol. 2, pp. 37-40, 1997.

[8] Allan Heydon and Marc Najork. Mercator: A scalable, extensible web crawler. World Wide Web Conference, 2(4):219-229, April 1999.

[9] Jenny Edwards, Kevin S.McCurley, and John A. Tomlin. An adaptive model for optimizing performance of an incremental web crawler. In Proceedings of the Tenth Conference on World Wide Web, pages 106113, Hong Kong, May 2001. Elsevier Science.

[10] Luciano Barbosa and Juliana Freire. Combining classifiers to identify online databases. In Proceedings of the 16th international conference on World Wide Web, pages 431-440. ACM, 2007.

[11] Mr. Anand Kolapkar , Prof. B. B. Gite, Secure Multimodal Authentication Using Watermarking,4April-2104.

[12] Mr. Anand Kolapkar , Prof. B. B. Gite,Application Research of MD5 Algorithm in LSB Watermarking, September, 2013. 